\title{
INVARIANT EINSTEIN METRICS ON GENERALIZED FLAG MANIFOLDS WITH TWO ISOTROPY SUMMANDS
}

\author{
ANDREAS ARVANITOYEORGOS and IOANNIS CHRYSIKOS ${ }^{凶}$
}

(Received 27 November 2008; accepted 6 October 2010)

Communicated by M. K. Murray

\begin{abstract}
Let $M=G / K$ be a generalized flag manifold, that is, an adjoint orbit of a compact, connected and semisimple Lie group $G$. We use a variational approach to find non-Kähler homogeneous Einstein metrics for flag manifolds with two isotropy summands. We also determine the nature of these Einstein metrics as critical points of the scalar curvature functional under fixed volume.
\end{abstract}

2010 Mathematics subject classification: primary 53C25; secondary 53C30, 22E46.

Keywords and phrases: Einstein manifold, homogeneous space, generalized flag manifold, isotropy representation, highest weight, Weyl's formula, bordered Hessian.

\section{Introduction}

A Riemannian manifold $(M, g)$ is called Einstein if the metric $g$ has constant Ricci curvature, that is, $\operatorname{Ric}_{g}=c g$, for some $c \in \mathbb{R}$. This equation, known as the Einstein equation, reduces to a system of nonlinear second-order partial differential equations, and general existence results are difficult to obtain. A common strategy for constructing Einstein metrics is to assume that $M$ is a homogeneous Riemannian manifold; this is the case when a Lie group $G$ of isometries acts transitively on $M$. Then the Einstein equation reduces to a more manageable system of (nonlinear) polynomial equations, which in some cases can be solved explicitly (see [Bes, NRS]).

If $(M, g)$ is a compact Riemannian manifold of volume one, then a result of Hilbert states that $g$ is Einstein if and only if $g$ is a critical point of the scalar curvature functional $T: \mathcal{M}_{1} \rightarrow \mathbb{R}$, given by

$$
T(g)=\int_{M} S(g) d \operatorname{vol}_{g}
$$

The authors were partially supported by C. Carathéodory grant number C.161 2007-10, University of Patras.

(C) 2011 Australian Mathematical Publishing Association Inc. 1446-7887/2011 \$16.00 
on the set $\mathcal{M}_{1}$ of Riemannian metrics of unit volume (see [Bes]). For a compact homogeneous Riemannian manifold $(M=G / K, g)$ of a compact connected Lie group $G$, one can show that the $G$-invariant Einstein metrics of volume one are precisely the critical points of $T$ restricted to $\mathcal{M}_{1}^{G}$, the set of $G$-invariant Riemannian metrics of volume one. This variational approach has led to several important existence and nonexistence results for homogeneous Einstein metrics (see, for example, [Bom, BWZ, DKe, WZ2]). For a detailed exposition on homogeneous Einstein manifolds and their classification problem we refer to Besse's book [Bes], and for more recent results to the surveys [LWa, NRS].

Let $G$ be a compact connected semisimple Lie group and let $\mathfrak{g}=T_{e} G$ be its Lie algebra. A generalized flag manifold is a (compact) homogeneous space of the form $M=G / C(S)$, where $C(S)$ is the centralizer of a torus $S$ in $G$. This condition can be reformulated as follows: $M$ is the orbit of an element $\gamma_{o} \in \mathfrak{g}$ under the adjoint action Ad $: G \times \mathfrak{g} \rightarrow \mathfrak{g}$, that is,

$$
M=\operatorname{Ad}(G) \gamma_{o}=\left\{\operatorname{Ad}(g) \gamma_{o}: g \in G\right\} \subseteq \mathfrak{g} .
$$

In fact, it can be shown that the stabilizer of this action $K=\left\{g \in G: \operatorname{Ad}(g) \gamma_{o}=\gamma_{o}\right\}$ is the centralizer of the torus $S_{\gamma_{o}}=\left\{\exp t \gamma_{o}: t \in \mathbb{R}\right\}^{-} \subseteq G$ generated by the one-parameter subgroup exp $t \gamma_{o}$ of $G$. In particular, $K$ is connected (see [Bes]). If $S_{\gamma_{o}}=T$ is a maximal torus in $G$, then $K=C\left(S_{\gamma_{o}}\right)=T$ and $M=G / T$ is called a full flag manifold. The classification of flag manifolds is based on painted Dynkin diagrams and can been found in several sources (see [Ale, AlA, BFR]). There is an infinite family for each of the classical Lie group types, and a finite number for each of the exceptional Lie groups. Note that in this work, following [AlA], we paint the Dynkin diagram in the opposite way to [BFR].

As we will see in Section 3, a flag manifold $M=G / C(S)$ can also be identified with a compact simply connected complex homogeneous space of the form $G^{\mathbb{C}} / P$ (also known as a complex flag manifold), where $P$ is a parabolic subgroup. Thus $M$ admits a finite number of invariant complex structures. In particular, for any such complex structure there is a unique $G$-invariant Kähler-Einstein metric. The problem of finding non-Kähler-Einstein metrics on generalized flag manifolds was first studied by Alekseevsky in [Ale]. For some of these spaces the standard metric is Einstein since they appear in the work of Wang and Ziller (see [WZ1]), where they classified all normal homogeneous Einstein manifolds. In [Kim], Kimura used the variational method of [WZ2] to find all $G$-invariant Einstein metrics for all flag manifolds for which the isotropy representation decomposes into three nonequivalent irreducible summands. In [Sak], Sakane gave an explicit expression for the Ricci tensor of full flag manifolds of classical Lie groups, and then by using the theory of Gröbner bases he proved the existence of non-Kähler homogeneous Einstein metrics. Finally, in [Arv], the first author found new $G$-invariant Einstein metrics on certain generalized flag manifolds with four isotropy summands, by using a Lie-theoretic description of the Ricci tensor.

In this paper we study generalized flag manifolds $M=G / K$ of a compact connected simple Lie group $G$, for which the isotropy representation decomposes into two 
TABLE 1. Generalized flag manifolds for which $m$ has two irreducible summands.

\begin{tabular}{ll}
\hline$G$ simple & $M=G / K$ where $\mathrm{m}=\mathrm{m}_{1} \oplus \mathrm{m}_{2}$ \\
\hline$B_{\ell}=\mathrm{SO}(2 \ell+1)$ & $\mathrm{SO}(2 \ell+1) / \mathrm{U}(p) \times \mathrm{SO}(2(\ell-p)+1)(2 \leq p \leq \ell)$ \\
$C_{\ell}=\mathrm{Sp}(\ell)$ & $\mathrm{Sp}(\ell) / \mathrm{U}(p) \times \mathrm{Sp}(\ell-p)(1 \leq p \leq \ell-1)$ \\
$D_{\ell}=\mathrm{SO}(2 \ell)$ & $\mathrm{SO}(2 \ell) / \mathrm{U}(p) \times \mathrm{SO}(2(\ell-p))(2 \leq p \leq \ell-2)$ \\
$G_{2}$ & $G_{2} / \mathrm{U}(2)\left(\mathrm{U}(2)\right.$ is represented by the short root of $\left.G_{2}\right)$ \\
$F_{4}$ & $F_{4} / \mathrm{SO}(7) \times \mathrm{U}(1)$ \\
& $F_{4} / \mathrm{Sp}(3) \times \mathrm{U}(1)$ \\
$E_{6}$ & $E_{6} / \mathrm{SU}(6) \times \mathrm{U}(1)$ \\
& $E_{6} / \mathrm{SU}(2) \times \mathrm{SU}(5) \times \mathrm{U}(1)$ \\
& $E_{7} / \mathrm{SU}(7) \times \mathrm{U}(1)$ \\
$E_{7}$ & $E_{7} / \mathrm{SU}(2) \times \mathrm{SO}(10) \times \mathrm{U}(1)$ \\
& $E_{7} / \mathrm{SO}(12) \times \mathrm{U}(1)$ \\
& $E_{8} / E_{7} \times \mathrm{U}(1)$ \\
$E_{8}$ & $E_{8} / \mathrm{SO}(14) \times \mathrm{U}(1)$ \\
\hline
\end{tabular}

nonequivalent irreducible submodules. Any such space admits a unique $G$-invariant complex structure and thus a unique $G$-invariant Kähler-Einstein metric. The authors classified these spaces in a recent paper $[\mathrm{ArC}]$ and proved that any such flag manifold is locally isomorphic to one of the spaces listed in Table 1.

Let $G$ be a simple compact Lie group and let $K$ be a connected and closed subgroup. In recent work [DKe], Dickinson and Kerr used [WZ2, Theorem 3.1] to count the number $\mathcal{E}(M)$ of Einstein metrics for all simply connected homogeneous spaces $M=G / K$, whose isotropy representation decomposes into two irreducible summands. For example, they proved that $\mathcal{E}(M)=2$ for any flag manifold $M=G / K$ for which $m$ is a sum of two irreducible submodules (see Table 1). In this work, we apply the variational method to find these two Einstein metrics. After this work was completed, the authors were informed by Sakane that solutions of the Einstein equation have also been obtained in unpublished work of Ohmura [Ohm], using the method of Riemannian submersions (see [Bes]).

The paper is organized as follows. In Section 2 we recall some facts about compact homogeneous spaces. In Section 3 we study the structure of a generalized flag manifold $M=G / K$ of a compact, connected, semisimple Lie group $G$. In Section 4 we recall how one can determine flag manifolds $M=G / K$ with $\mathfrak{m}=\mathfrak{m}_{1} \oplus \mathfrak{m}_{2}$ and we make a observation on the computation of the dimensions $d_{i}=\operatorname{dim} m_{i}$ of the irreducible submodules. In Section 5 we consider a general $G$-invariant Riemannian metric $g=\left.x_{1}(-B)\right|_{\mathfrak{m}_{1}}+\left.x_{2}(-B)\right|_{\mathfrak{m}_{2}}$ on $M=G / K$, and by applying a variational method, we solve the Einstein equation. We prove the following theorem. 
THeorem 1.1. Let $M=G / K$ be a generalized flag manifold with two irreducible isotropy summands, that is, $\mathfrak{m}=\mathrm{m}_{1} \oplus \mathfrak{m}_{2}$, and set $d_{i}=\operatorname{dim} \mathfrak{m}_{i}$. Then, up to scale, $M$ admits two G-invariant Einstein metrics. One, given by $x_{1}=1$ and $x_{2}=2$, is Kähler and the other, given by $x_{1}=1$ and $x_{2}=4 d_{2} /\left(d_{1}+2 d_{2}\right)$, is not.

Next we compute the bordered Hessian of the scalar curvature functional with the volume-one constraint condition and characterize the nature of the solutions (as critical points) obtained in Theorem 1.1. In particular, we show the following result.

Theorem 1.2. Let $M=G / K$ be a generalized flag manifold with two isotropy summands. Then the two G-invariant Einstein metrics on M, obtained in Theorem 1.1, are both local minima of the scalar curvature functional restricted to the space of G-invariant metrics of volume one.

\section{Preliminaries}

Let $M=G / K$ be a homogeneous space, where $G$ is a compact connected and semisimple Lie group and $K$ is a closed subgroup of $G$. We denote by $o=e K$ the identity coset of $G / K$ and by $\mathfrak{g}$ and $\mathfrak{f}$ the Lie algebras of $G$ and $K$. Since the Lie group $G$ is semisimple and compact, the $\operatorname{Ad}(K)$-invariant Killing form $B(X, Y)=\operatorname{tr}(\operatorname{ad}(X) \circ \operatorname{ad}(Y))$ is negative definite. Consider the reductive decomposition $\mathfrak{g}=\mathfrak{f} \oplus \mathfrak{m}$ where $\mathfrak{m}=\mathfrak{f}^{\perp}$ (with respect to $-B$ ) and $\operatorname{Ad}(K) \mathfrak{m} \subseteq \mathfrak{m}$. Then there is a natural isomorphism between the tangent space $T_{o} M$ and the linear subspace $\mathrm{m}$. Also, the isotropy representation $\chi: K \rightarrow \operatorname{Aut}(\mathfrak{m})$ of $K$ is equivalent to the adjoint representation of $K$ restricted on $\mathrm{m}$, that is, $\chi(K)=\left.\operatorname{Ad}^{K}\right|_{\mathfrak{m}}$. Therefore a $G$-invariant metric $g$ on $G / K$ is determined by an $\operatorname{Ad}(K)$-invariant inner product on $\mathfrak{m}$ (see [KoN, NRS]).

Let $Q(\cdot, \cdot)$ be an $\operatorname{Ad}(K)$-invariant inner product on $m$ (which is a multiple of the Killing form), and take a $Q$-orthogonal decomposition

$$
\mathfrak{m}=\mathfrak{m}_{1} \oplus \cdots \oplus \mathfrak{m}_{q}
$$

of $m$ into its $\operatorname{Ad}(K)$-irreducible submodules. For such a decomposition, there is a family of $G$-invariant metrics on $G / K($ or $\operatorname{Ad}(K)$-invariant inner products $\langle\cdot, \cdot\rangle$ on $\mathfrak{m})$, given by

$$
\langle\cdot, \cdot\rangle=\left.x_{1} Q\right|_{\mathfrak{m}_{1}}+\cdots+\left.x_{q} Q\right|_{\mathfrak{m}_{q}},
$$

where $\left(x_{1}, \ldots, x_{q}\right) \in \mathbb{R}_{+}^{q}$. This follows immediately from Schur's lemma, after a simultaneous diagonalization of the form $\langle\cdot, \cdot\rangle$ and $Q$. If the representations $m_{i}$ and $\mathrm{m}_{j}$ are pairwise nonequivalent whenever $i \neq j$, then the decomposition (2.1) is unique up to order and any $G$-invariant metric on $G / K$ has the form (2.2). If the modules $\mathfrak{m}_{i}$ and $\mathfrak{m}_{j}$ are equivalent for some $i$ and $j$, then $\left\langle\mathfrak{m}_{i}, \mathfrak{m}_{j}\right\rangle$ does not necessarily vanish. In the rest of this paper, we assume that $\mathfrak{m}_{i} \neq \mathrm{m}_{j}$ whenever $i \neq j$.

Since $G$ is compact and $K$ is a closed subgroup of $G$, the homogeneous space $G / K$ is compact. Thus we can apply the variational method to find $G$-invariant Einstein metrics on $G / K$. Let $\mathcal{M}_{1}^{G}$ denote the set of all $G$-invariant metrics on $G / K$ of 
volume one. Note that a metric $g=\langle\cdot, \cdot\rangle$ as in (2.2) belongs to $\mathcal{M}_{1}^{G}$ if and only if $\prod_{i=1}^{q} x_{i}^{d_{i}}=1$, where $d_{i}=\operatorname{dim} m_{i}$. The space $\mathcal{M}_{1}^{G}$ is a flat Riemannian manifold whose dimension is equal to the number of irreducible summands, since the isotropy representation of $G / K$ consists of pairwise nonequivalent irreducible representations (see [BWZ, p. 693]). Let $S=S(g)$ denote the scalar curvature of $g=\langle\cdot, \cdot\rangle$. Then $T(g)=S(g)$ on $\mathcal{M}_{1}^{G}$, since $S=S(g)$ is a constant function, and the $G$-invariant Einstein metrics of volume one on $G / K$ are precisely the critical points of the restriction

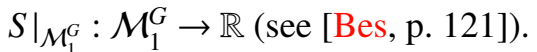

Following [WZ2], given a decomposition (2.1) we define triples [ijk], where $i, j, k \in\{1, \ldots, q\}$ as follows: $[i j k]=\sum_{\alpha, \beta, \gamma}\left(A_{\alpha \beta}^{\gamma}\right)^{2}$, where $A_{\alpha \beta}^{\gamma}=Q\left(\left[X_{\alpha}, X_{\beta}\right], X_{\gamma}\right)$ so that $\left[X_{\alpha}, X_{\beta}\right]=\sum_{\gamma} A_{\alpha \beta}^{\gamma} X_{\gamma}$. Here by $X_{\alpha}, X_{\beta}, X_{\gamma}$ we denote $Q$-orthogonal bases of the submodules $\mathrm{m}_{i}, \mathrm{~m}_{j}$ and $\mathrm{m}_{k}$ respectively. Note that $[i j k]$ is nonnegative and symmetric in $i, j$ and $k$.

Proposition 2.1 (See [WZ2]). The scalar curvature of $(M=G / K, g=\langle\cdot, \cdot\rangle)$ is given by

$$
S=\frac{1}{2} \sum_{i=1}^{q} \frac{d_{i} f_{i}}{x_{i}}-\frac{1}{4} \sum_{i, j, k}[i j k] \frac{x_{k}}{x_{i} x_{j}}
$$

where the numbers $f_{i}$ are defined by $-\left.B\right|_{\mathfrak{m}_{i}}=\left.f_{i} Q\right|_{\mathfrak{m}_{i}}$ when $i=1, \ldots, q$.

\section{Generalized flag manifolds and painted Dynkin diagrams}

3.1. The algebraic description of a flag manifold. Let $G$ be a compact simple Lie group with trivial center and let $\gamma_{o}$ be an element of its Lie algebra $\mathfrak{g}$. In this section we will give the Lie-theoretic description of the generalized flag manifold $M=\operatorname{Ad}(G) \gamma_{o}=G / K$, where $K=C(S)$ is the centralizer of a torus $S$ in $G$. Choose a maximal torus $T$ in $G$ which contains the torus $S$ and let $\mathfrak{h}$ be the Lie algebra of $T$. Then $S \subseteq T \subseteq C(S)=K$ and $T$ is also a maximal torus for the isotropy subgroup $K$. Thus $G$ and $K$ satisfy rank $G=\operatorname{rank} K$. We will denote by $\mathfrak{g}^{\mathbb{C}}$ and $\mathfrak{h}^{\mathbb{C}}$ the complexifications of $\mathfrak{g}$ and $\mathfrak{h}$ respectively.

Let $\mathfrak{g}^{\mathbb{C}}=\mathfrak{h}^{\mathbb{C}} \oplus \sum_{\alpha \in R} \mathfrak{g}_{\alpha}^{\mathbb{C}}$ be the root space decomposition of $\mathfrak{g}^{\mathbb{C}}$ with respect to the Cartan subalgebra $\mathfrak{h}^{\mathbb{C}}$. Here $R \subseteq\left(\mathfrak{h}^{\mathbb{C}}\right)^{*} \backslash\{0\}$ is the root system of $\mathfrak{g}^{\mathbb{C}}$ and $\mathfrak{g}_{\alpha}^{\mathbb{C}}$ is the root space associated to a root $\alpha$. Let $\Pi=\left\{\alpha_{1}, \ldots, \alpha_{\ell}\right\}$ be a system of simple roots for $R$, where $\operatorname{dim} \mathfrak{h}^{\mathbb{C}}=\ell$. Fix a lexicographic ordering on $\left(\mathfrak{h}^{\mathbb{C}}\right)^{*}$ and let $R^{+}$be the set of positive roots. For any $\alpha \in R$ choose root vectors $E_{\alpha} \in \mathfrak{g}_{\alpha}^{\mathbb{C}}$ such that $B\left(E_{\alpha}, E_{-\alpha}\right)=-1$ and $\left[E_{\alpha}, E_{-\alpha}\right]=-H_{\alpha}$, where $H_{\alpha} \in \mathfrak{h}^{\mathbb{C}}$ is determined by the condition $B\left(H, H_{\alpha}\right)=\alpha(H)$ for all $H \in \mathfrak{h}^{\mathbb{C}}$. In this way we obtain a natural isomorphism between $\mathfrak{h}^{\mathbb{C}}$ and the dual space $\left(\mathfrak{h}^{\mathbb{C}}\right)^{*}$. The normalized vectors $E_{\alpha}$ are such that

$$
\left[E_{\alpha}, E_{\beta}\right]= \begin{cases}N_{\alpha, \beta} E_{\alpha+\beta} & \text { if } \alpha, \beta, \alpha+\beta \in R, \\ 0 & \text { if } \alpha, \beta \in R \text { and } \alpha+\beta \notin R,\end{cases}
$$


where $N_{\alpha, \beta}=N_{-\alpha,-\beta} \in \mathbb{R}$ when $\alpha, \beta \in R$. Then the real Lie algebra $\mathfrak{g}$ is given by

$$
\mathfrak{g}=\mathfrak{h} \oplus \sum_{\alpha \in R^{+}}\left(\mathbb{R} A_{\alpha}+\mathbb{R} B_{\alpha}\right),
$$

where $A_{\alpha}=E_{\alpha}+E_{-\alpha}$ and $B_{\alpha}=\sqrt{-1}\left(E_{\alpha}-E_{-\alpha}\right)$ when $\alpha \in R^{+}$(see [Hel]).

As $\mathfrak{h}^{\mathbb{C}} \subseteq \mathfrak{f}^{\mathbb{C}} \subseteq \mathfrak{g}^{\mathbb{C}}$, there is a closed subsystem $R_{K}$ of $R$ such that $\mathfrak{f}^{\mathbb{C}}=\mathfrak{h}^{\mathbb{C}} \oplus \sum_{\alpha \in R_{K}} \mathfrak{g}_{\alpha}^{\mathbb{C}}$. In particular, we can always find a subset $\Pi_{K} \subseteq \Pi$ such that

$$
R_{K}=R \cap\left\langle\Pi_{K}\right\rangle=\left\{\beta \in R: \beta=\sum_{\alpha_{i} \in \Pi_{K}} k_{i} \alpha_{i}, k_{i} \in \mathbb{Z}\right\},
$$

where $\left\langle\Pi_{K}\right\rangle$ is the space of roots generated by $\Pi_{K}$ with integer coefficients. Note that $\mathfrak{f}^{\mathbb{C}}$ is a maximal reductive subalgebra of $\mathfrak{g}^{\mathbb{C}}$ and thus the decomposition $\mathfrak{f}^{\mathbb{C}}=3\left(\mathfrak{f}^{\mathbb{C}}\right) \oplus \mathfrak{f}_{\mathrm{ss}}^{\mathbb{C}}$ holds. Here $z\left(\mathfrak{f}^{\mathbb{C}}\right)$ is the center of $\mathfrak{f}^{\mathbb{C}}$ and $\mathfrak{f}_{\mathrm{ss}}^{\mathbb{C}}=\left[\mathfrak{f}^{\mathbb{C}}, \mathfrak{f}^{\mathbb{C}}\right]$ is its semisimple part. In fact, the subset $\Pi_{K} \subseteq \Pi$ is a system of simple roots for the complex semisimple Lie algebra $\mathfrak{f}_{\mathrm{ss}}^{\mathbb{C}}$ and $R_{K}$ is the associated root system (see [Kim]). As usual, we set $R_{K}^{+}=R^{+} \cap\left\langle\Pi_{K}\right\rangle$, $R_{M}=R \backslash R_{K}$ and $R_{M}^{+}=R^{+} \backslash R_{K}^{+}$. Then it can be proved that $\mathfrak{p}$, given by

$$
\mathfrak{p}=\mathfrak{h}^{\mathbb{C}} \oplus \sum_{\alpha \in R_{K}} \mathfrak{g}_{\alpha}^{\mathbb{C}} \oplus \sum_{\alpha \in R_{M}^{+}} \mathfrak{g}_{\alpha}^{\mathbb{C}},
$$

is a parabolic subalgebra of $\mathfrak{g}^{\mathbb{C}}$ (see [Ale]).

Let $G^{\mathbb{C}}$ be the simply connected complex simple Lie group whose Lie algebra is $\mathfrak{g}^{\mathbb{C}}$ and let $P$ be the parabolic subgroup of $G^{\mathbb{C}}$ generated by $\mathfrak{p}$. The homogeneous space $G^{\mathbb{C}} / P$ is a compact, simply connected, complex manifold on which $G$ acts transitively (see [Wol]). In particular, the isotropy subgroup $K=G \cap P$ is a connected closed subgroup of $G$ which is identified with the centralizer of a torus $S$ in $G$. Thus we obtain the diffeomorphism $G^{\mathbb{C}} / P \cong G / K$. The complex representation $G^{\mathbb{C}} / P$ implies that $M$ is a complex manifold, endowed with a $G$-invariant complex structure. The real representation $G / K=G / C(S)$ implies that $M$ is Kähler (see [Bor, Wan]). In general, $M$ admits several $G$-invariant Kählerian structures (see [Ale, BoH]).

We recall briefly the definition of painted Dynkin diagrams, following [AlA]. Take $\Pi_{M}=\Pi \backslash \Pi_{K}$ such that $\Pi=\Pi_{K} \sqcup \Pi_{M}$. Then all information contained in the pair $\left(\Pi, \Pi_{K}\right.$ ) can be presented graphically by the painted Dynkin diagram of $M=G / K$.

Definition 3.1. Let $\Gamma=\Gamma(\Pi)$ be the Dynkin diagram corresponding to the system of simple roots $\Pi$. By painting black the nodes of $\Gamma$ corresponding to $\Pi_{M}=\Pi \backslash \Pi_{K}$, we obtain the painted Dynkin diagram $\Gamma_{\Pi_{M}}$ of the flag manifold $G / K$.

The subdiagram of white nodes with the connecting lines between them determines the subsystem $\Pi_{K}$ and hence the semisimple part of the Lie algebra of $K$. Further, each black simple root, that is, each element of $\Pi_{M}$, gives rise to a $\mathrm{U}(1)$-component, and hence determines $K$. Note that we often make use of the diffeomorphism $\mathrm{SU}(n) \times \mathrm{U}(1) \cong \mathrm{U}(n)$. By using appropriate rules to determine whether different painted Dynkin diagrams define isomorphic flag manifolds (see, for example, [AlA]), we can obtain all flag manifolds $G / K$ of a simple Lie group $G$. 
3.2. Flag manifolds with second Betti number one. We will now study the isotropy representation for generalized flag manifolds $M=G / K$ with second Betti number $b_{2}(M)$ equal to one. Recall (see [BoH, p. 507]) that if $M=G / K$ is a generalized flag manifold defined by a pair $\left(\Pi, \Pi_{K}\right)$, then

$$
b_{2}(M)=\operatorname{card}\left(\Pi \backslash \Pi_{K}\right)=\operatorname{card}\left(\Pi_{M}\right) .
$$

Typical examples of such spaces are the isotropy irreducible compact Hermitian symmetric spaces. In Section 4, we will see that all flag manifolds with two isotropy summands have second Betti number equal to one.

Flag manifolds $M=G / K$ with $b_{2}(M)=1$ are obtained by painting black only one simple root in the Dynkin diagram of $G$, that is, $\Pi \backslash \Pi_{K}=\Pi_{M}=\left\{\alpha_{p}\right\}$ for some fixed index $p$ between 1 and $\ell$. Let $\mathfrak{g}=\mathfrak{f} \oplus \mathrm{m}$ be a $(-B)$-orthogonal reductive decomposition of $\mathfrak{g}$. Due to the splitting $R_{M}^{+}=R^{+} \backslash R_{K}^{+}$, the $\operatorname{Ad}(K)$-invariant subspace $\mathrm{m}$ is given by $\mathrm{m}=\sum_{\alpha \in R_{M}^{+}}\left(\mathbb{R} A_{\alpha}+\mathbb{R} B_{\alpha}\right)$. For a nonnegative integer $n$, we set

$$
R^{+}\left(\alpha_{i}, n\right)=\left\{\alpha \in R^{+}: \alpha=\sum_{j=1}^{\ell} m_{j} \alpha_{j} \in R^{+}, m_{p}=n\right\},
$$

and define $\operatorname{Ad}(K)$-invariant subspaces $\mathfrak{m}_{n}$ of $\mathfrak{g}$ by $\mathfrak{m}_{n}=\sum_{\alpha \in R^{+}\left(\alpha_{i}, n\right)}\left(\mathbb{R} A_{\alpha}+\mathbb{R} B_{\alpha}\right)$. Put $q=\max \left\{m_{p}: \alpha=\sum_{j=1}^{\ell} m_{j} \alpha_{j} \in R^{+}\right\}$. Then $R_{M}^{+}=\bigcup_{n=1}^{q} R^{+}\left(\alpha_{i}, n\right)$ and

$$
\mathfrak{m}=\sum_{n=1}^{q} \mathfrak{m}_{n}
$$

For simplicity, we set $\mathrm{m}_{0}=\mathfrak{f}$. Then when $n, m \in\{1, \ldots, q\}$, we have (see [Ikw, Ith])

$$
\left[\mathfrak{f}, \mathfrak{m}_{n}\right] \subseteq \mathfrak{m}_{n}, \quad\left[\mathfrak{m}_{n}, \mathfrak{m}_{m}\right] \subseteq \mathfrak{m}_{n+m}+\mathfrak{m}_{|n-m|}, \quad\left[\mathfrak{m}_{n}, \mathfrak{m}_{n}\right] \subseteq \mathfrak{l} \oplus \mathfrak{m}_{2 n} .
$$

Note that the $\mathrm{m}_{n}$ are irreducible as $\operatorname{Ad}(K)$-modules. Also, they are nonequivalent to each other (see [Kim]). Thus (3.1) defines an irreducible decomposition of $m$, and any $G$-invariant metric on $G / K$ is given by (2.2). The following proposition describes the unique Kähler-Einstein metric on $M=G / K$.

Proposition 3.2 (See [Bor, BoH]). Let $M=G / K$ be a flag manifold for which $b_{2}(M)=$ 1. Assume that (3.1) defines a decomposition of $m$ into q irreducible submodules. Then $M$ admits a unique G-invariant Kähler-Einstein metric given by

$$
g=\langle\cdot, \cdot\rangle=\left.1(-B)\right|_{\mathfrak{m}_{1}}+\left.2(-B)\right|_{\mathfrak{m}_{2}}+\cdots+\left.q(-B)\right|_{\mathfrak{m}_{q}} .
$$

\section{Flag manifolds with two isotropy summands}

4.1. The classification. Let $G$ be a compact, connected, simple Lie group $G$ and let $\Pi=\left\{\alpha_{1}, \ldots, \alpha_{\ell}\right\}$ be a system of simple roots of the associated root system $R$. In [ArC], the authors proved that flag manifolds $M=G / K$ with $\mathfrak{m}=\mathfrak{m}_{1} \oplus \mathfrak{m}_{2}$ are in a one-toone correspondence with pairs $\left(\Pi, \Pi_{K}\right)$ of the form $\Pi \backslash \Pi_{K}=\Pi_{M}=\left\{\alpha_{p}\right\}$ for which the height of the simple root $\alpha_{p}$ is 2 . Recall that the height of a simple root $\alpha_{p} \in \Pi$ is the 
positive integer $c_{p}$ in the expression for the highest root $\tilde{a}=\sum_{i=1}^{\ell} c_{i} \alpha_{i} \in R$ in terms of the elements of $\Pi$. We describe all these suitable pairs $\left(\Pi, \Pi_{K}\right)$ in Table 2 . Indeed, note that any flag manifold $M=G / K$ with two isotropy summands is such that $b_{2}(M)=1$. Thus, with the notation of Section 3.2, it is sufficient to take $R^{+}\left(\alpha_{i}, n\right)=0$ where $n \geq 3$, that is, $n \in\{1,2\}$ and $q=c_{p}=2$.

4.2. A note on the dimensions of the irreducible submodules. For the flag manifolds $M=G / K$ corresponding to a simple Lie group $G$ of type $B_{\ell}, C_{\ell}$ or $D_{\ell}$, the simplest method to compute the dimensions of the irreducible submodules is a straightforward computation of the isotropy representation $\chi$, using the relation $\left.\mathrm{Ad}^{G}\right|_{K}=\operatorname{Ad}^{K} \oplus \chi($ see $[\mathrm{WZ1} 1)$.

ExAmple 4.1. We consider the flag manifold $M=\operatorname{Sp}(\ell) / \mathrm{U}(p) \times \operatorname{Sp}(\ell-p)$. Results are obtained similarly for the flag manifolds $\mathrm{SO}(2 \ell+1) / \mathrm{U}(p) \times \mathrm{SO}(2(\ell-p)+1)$ and $\mathrm{SO}(2 \ell) / \mathrm{U}(p) \times \mathrm{SO}(2(\ell-p))$. Let $\mu_{p}: \mathrm{U}(p) \rightarrow \operatorname{Aut}\left(\mathbb{C}^{p}\right)$ and $v_{\ell}: \operatorname{Sp}(\ell) \rightarrow \operatorname{Aut}\left(\mathbb{C}^{2 \ell}\right)$ be the standard representations of the Lie groups $\mathrm{U}(p)$ and $\mathrm{Sp}(\ell)$. It is known (see [WZ1]) that $\mathrm{Ad}^{\mathrm{U}(p)} \otimes \mathbb{C}=\mu_{p} \otimes_{\mathbb{C}} \overline{\mu_{p}}$ and $\mathrm{Ad}^{\mathrm{Sp}(\ell)} \otimes \mathbb{C}=\mathrm{S}^{2} v_{\ell}$, where $\mathrm{S}^{2}$ is the second symmetric power of $\mathbb{C}^{2 \ell}$. Then

$$
\begin{aligned}
\operatorname{Ad}^{\mathrm{Sp}(\ell)} \otimes \mathbb{C}_{\mathrm{U}(p) \times \mathrm{Sp}(\ell-p)}= & \mathrm{S}^{2}\left(\left.v_{\ell}\right|_{\mathrm{U}(p) \times \mathrm{Sp}(\ell-p)}\right) \\
= & \mathrm{S}^{2}\left(\mu_{p} \oplus \bar{\mu}_{p} \oplus v_{\ell-p}\right) \\
= & \mathrm{S}^{2} \mu_{p} \oplus \mathrm{S}^{2} \bar{\mu}_{p} \oplus \mathrm{S}^{2} v_{\ell-p} \oplus\left(\mu_{p} \otimes \bar{\mu}_{p}\right) \\
& \oplus\left(\mu_{p} \otimes v_{\ell-p}\right) \oplus\left(\bar{\mu}_{p} \otimes v_{\ell-p}\right) .
\end{aligned}
$$

The term $\mathrm{S}^{2} v_{\ell-p}$ corresponds to the complexified adjoint representation of $\operatorname{Sp}(\ell-p)$ and the term $\mu_{p} \otimes \bar{\mu}_{p}$ corresponds to the complexified adjoint representation of $\mathrm{U}(p)$. It follows that the complexified isotropy representation of $M$ is given by $\left(\mu_{p} \otimes v_{\ell-p}\right) \oplus\left(\bar{\mu}_{p} \otimes v_{\ell-p}\right) \oplus S^{2} \mu_{p} \oplus S^{2} \bar{\mu}_{p}$. This is the direct sum of four complex $\operatorname{ad}\left(\mathfrak{f}^{\mathbb{C}}\right)$-invariant nonequivalent submodules of dimensions $2 p(\ell-p), 2 p(\ell-p),\left(\begin{array}{c}p+1 \\ 2\end{array}\right)$ and $\left(\begin{array}{c}p+1 \\ 2\end{array}\right)$ respectively. The representations $\bar{\mu}_{p} \otimes v_{\ell-p}$ and $\mu_{p} \otimes v_{\ell-p}$ are conjugate to each other, as are $S^{2} \bar{\mu}_{p}$ and $S^{2} \mu_{p}$. Thus $m$ decomposes into a direct sum of two real irreducible submodules $m_{1}$ and $m_{2}$ of dimensions $4 p(\ell-p)$ and $p(p+1)$.

In order to compute the dimensions of $m_{1}$ and $m_{2}$ for the exceptional flag manifolds, we apply Weyl's dimension formula for irreducible representations (see [GOV, Hel]). For such a procedure one can use also the computer program on page 104 of the $\mathrm{LiE}$ manual (see http://www-math. univ-poitiers.fr/ maavl/LiE/).

Let $\mathfrak{g}$ be a complex semisimple Lie algebra with Cartan subalgebra $\mathfrak{h}$, and let $\mathfrak{g}=\mathfrak{h} \oplus \sum_{\alpha \in R} \mathfrak{g}_{\alpha}$ be the corresponding root space decomposition. Let $\Pi=\left\{\alpha_{1}, \ldots, \alpha_{\ell}\right\}$ be a system of simple roots for $R$ and let $R^{+}$be the set of positive roots. Consider the fundamental weights $\Lambda_{1}, \ldots, \Lambda_{\ell}$, that is, the linear forms defined by the equation $2\left(\Lambda_{i}, \alpha_{j}\right) /\left(\alpha_{j}, \alpha_{j}\right)=\delta_{i j}$ whenever $i, j=1, \ldots, \ell$. It is well known that $\left\{\Lambda_{i}\right\}$ is a basis of $\mathfrak{h}^{*}$, dual to the basis $\left\{h_{\alpha_{i}}=2 \alpha_{i} /\left(\alpha_{i}, \alpha_{i}\right)\right\}$ of $\mathfrak{h}$. Note that if $A=\left(a_{i j}\right)=$ $\left(2\left(\alpha_{i}, \alpha_{j}\right) /\left(\alpha_{i}, \alpha_{i}\right)\right)$ is the Cartan matrix of $\mathfrak{g}$ (with respect to $\left.\Pi\right)$, then the relation 
TABLE 2. Painted Dynkin diagrams of flag manifolds $M=G / K$ for which $m$ is reducible.

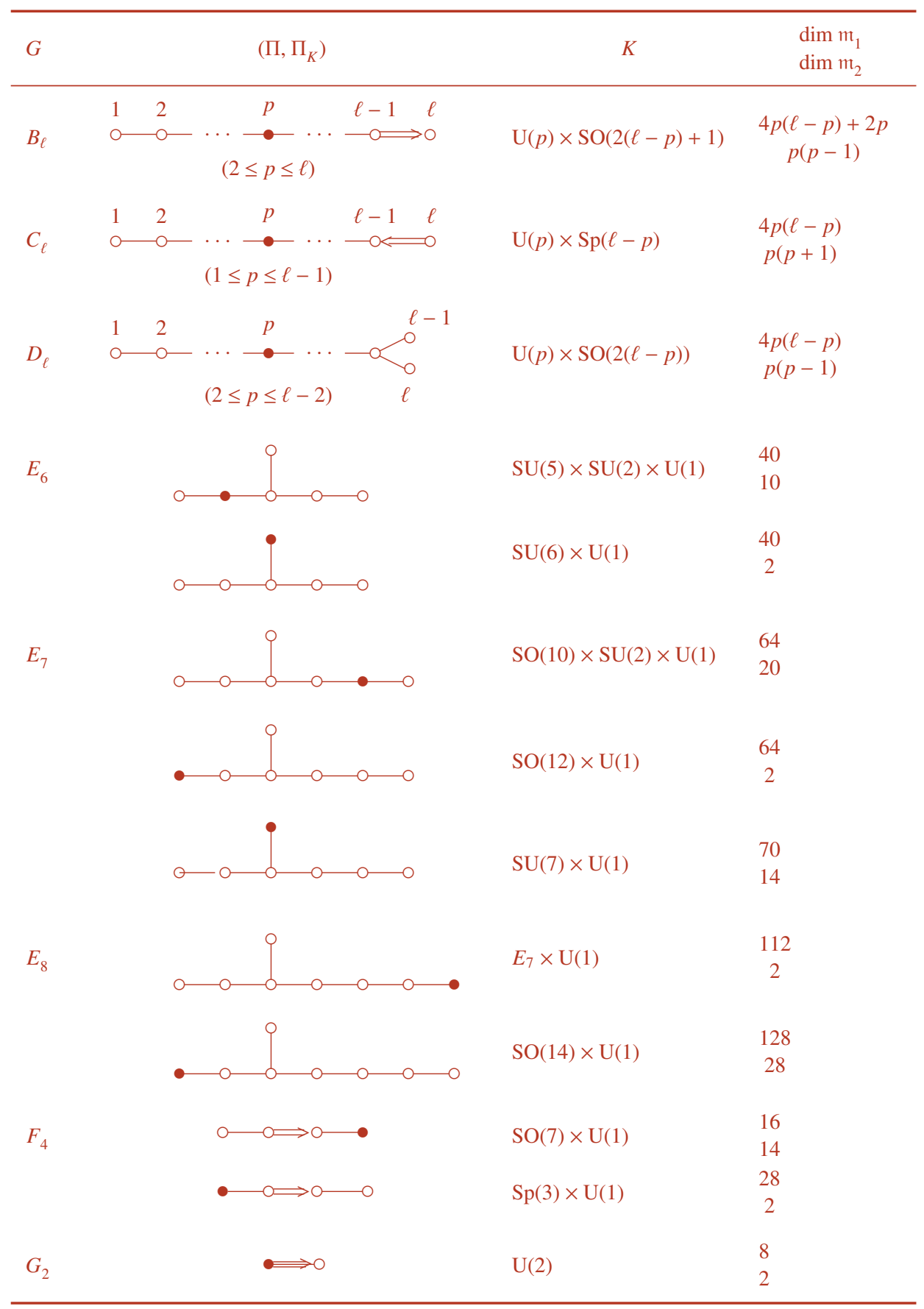


between the simple roots and the fundamental weights is given by $\alpha_{i}=\sum_{j=1}^{\ell} a_{j i} \Lambda_{j}$ (see $[\mathrm{GOV}])$.

Proposition 4.2 (See [GOV]). Let $\rho_{\lambda}: \mathfrak{g} \rightarrow \operatorname{End}(V)$ be the finite-dimensional representation of $\mathfrak{g}$ on the complex vector space $V$ with highest weight $\lambda$. Then

$$
\operatorname{dim}_{\mathbb{C}} \rho_{\lambda}=\prod_{\alpha \in R^{+}}\left(1+\frac{(\lambda, \alpha)}{(\delta, \alpha)}\right),
$$

where the weight $\delta$ is given by $\delta=(1 / 2) \sum_{\alpha \in R^{+}} \alpha=\sum_{i=1}^{\ell} \Lambda_{i}$.

Now let $M=G / K$ be a generalized flag manifold such that $\mathrm{m}=\mathrm{m}_{1} \oplus \mathrm{m}_{2}$, determined by a pair $\left(\Pi, \Pi_{K}\right)$. Since $m_{1}$ and $m_{2}$ are $\operatorname{Ad}(K)$-submodules, Weyl's formula takes the form

$$
\operatorname{dim}_{\mathbb{C}} \mathfrak{m}_{i}=\prod_{\alpha \in R_{K}^{+}}\left(1+\frac{\left(\lambda_{i}, \alpha\right)}{\left(\delta_{K}, \alpha\right)}\right),
$$

where $\lambda_{i}$ is the highest weight of the submodule $m_{i}$ when $i=1,2$, and $R_{K}^{+}=R^{+} \cap\left\langle\Pi_{K}\right\rangle$ is the positive root system of the semisimple part of the isotropy subgroup $K$ and

$$
\delta_{K}=\frac{1}{2} \sum_{\beta \in R_{K}^{+}} \beta .
$$

ExAmple 4.3. We consider the exceptional flag manifold $G / K=G_{2} / \mathrm{U}(2)$. The other cases are similar. For the root system of $G_{2}$, we use the notation of [AlA]. We fix a system of simple roots $\Pi=\left\{\alpha_{1}, \alpha_{2}\right\}=\left\{e_{2}-e_{3},-e_{2}\right\}$, and let

$$
R^{+}=\left\{\alpha_{1}, \alpha_{2}, \alpha_{1}+\alpha_{2}, \alpha_{1}+2 \alpha_{2}, \alpha_{1}+3 \alpha_{2}, 2 \alpha_{1}+3 \alpha_{2}\right\}
$$

Then $\left(\alpha_{1}, \alpha_{1}\right)=2$ and $\left(\alpha_{2}, \alpha_{2}\right)=2 / 3$. The highest root $\tilde{\alpha}$ is given by $\tilde{\alpha}=2 \alpha_{1}+3 \alpha_{2}$. The Cartan matrix $A=\left(a_{i j}\right)$ of $G_{2}$ with respect to $\Pi$ is given by

$$
A=\left(\begin{array}{rr}
2 & -1 \\
-3 & 2
\end{array}\right)
$$

The painted Dynkin diagram

$$
\stackrel{\alpha_{1}}{\rightleftharpoons} \alpha_{2}
$$

determines the generalized flag manifold $G_{2} / \mathrm{U}(2)$, where $\mathrm{U}(2)$ is represented by the short root $\alpha_{2}$. Thus $R_{K}^{+}=\left\{\alpha_{2}\right\}$. The highest weights of the irreducible $\operatorname{Ad}(K)$ submodules $m_{1}$ and $m_{2}$ are given by $\lambda_{1}=\alpha_{1}+3 \alpha_{2}$ and $\lambda_{2}=\tilde{\alpha}$ respectively. By using the transpose of the Cartan matrix, we obtain $\alpha_{1}=2 \Lambda_{1}-3 \Lambda_{2}$ and $\alpha_{2}=-\Lambda_{1}+3 \Lambda_{2}$, where $\Lambda_{1}, \Lambda_{2}$ are the fundamental weights of $G_{2}$. Thus $\lambda_{1}=-\Lambda_{1}+3 \Lambda_{2}$ and $\lambda_{2}=\Lambda_{1}$. Now we can use Weyl's formula, and deduce that $\operatorname{dim}_{\mathbb{C}} m_{1}=(1+(3 / 1))=4$ and $\operatorname{dim}_{\mathbb{C}} \mathfrak{m}_{2}=1$, therefore $\operatorname{dim}_{\mathbb{R}} \mathfrak{m}_{1}=8$ and $\operatorname{dim}_{\mathbb{R}} \mathfrak{m}_{2}=2$. 


\section{Homogeneous Einstein metrics}

5.1. The variational method. Let $M=G / K$ be a generalized flag manifold with two isotropy summands. Set $d_{1}=\operatorname{dim} \mathrm{m}_{1}$ and $d_{2}=\operatorname{dim} \mathrm{m}_{2}$. We will apply the variational method for a $G$-invariant metric $g=\langle\cdot, \cdot\rangle$ on $M$ to prove Theorem 1.1.

Proof of Theorem 1.1. First, we will use Proposition 2.1 to compute the scalar curvature of $(M=G / K, g=\langle\cdot, \cdot\rangle)$. According to (2.2), the $G$-invariant metric $g=\langle\cdot, \cdot\rangle$ has the form

$$
\langle\cdot, \cdot\rangle=\left.x_{1}(-B)\right|_{\mathfrak{m}_{1}}+\left.x_{2}(-B)\right|_{\mathfrak{m}_{2}},
$$

where $\left(x_{1}, x_{2}\right) \in \mathbb{R}_{+}^{2}$. Thus the space $\mathcal{M}_{1}^{G}$ is a two-dimensional flat Riemannian manifold.

Since $G$ is simple, $f_{i}=1$ when $i=1,2$. In order to apply Proposition 2.1, we need to find the triples $[i j k]$, where $i, j, k \in\{1,2\}$. Since $[i j k]$ is symmetric in its entries, $[111]=[222]=0$. From (3.2), we obtain $\left[\mathfrak{m}_{1}, \mathfrak{m}_{2}\right] \subseteq \mathfrak{m}_{1},\left[\mathfrak{m}_{1}, \mathfrak{m}_{1}\right] \subseteq \mathfrak{m}_{2} \oplus \mathfrak{f}$ and $\left[\mathfrak{m}_{2}, \mathfrak{m}_{2}\right] \subseteq \mathfrak{f}$. Thus $[221]=[212]=[122]=0$ and the only nonzero triples are $t=$ $[112]=[211]=[121]$. By formula (2.3), we easily conclude that the scalar curvature of $(M=G / K, g=\langle\cdot, \cdot\rangle)$ is given by

$$
S(g)=\frac{1}{2}\left(\frac{d_{1}}{x_{1}}+\frac{d_{2}}{x_{2}}\right)-\frac{1}{4}\left(t \frac{x_{2}}{x_{1}^{2}}+2 t \frac{1}{x_{2}}\right) .
$$

Let $V(g)=x_{1}^{d_{1}} x_{2}^{d_{2}}$ be the volume of the metric (5.1). In order to determine the $G$-invariant Einstein metrics of $G / K$ subject to the constraint $V(g)=1$, we need to study the critical points of the restricted scalar curvature $\left.S\right|_{\mathcal{M}_{1}^{G}}$. According to the Lagrange multiplier method, a metric $g=\left(x_{1}, x_{2}\right) \in \mathcal{M}_{1}^{G}$ is a critical point of $\left.S\right|_{\mathcal{M}_{1}^{G}}$ if and only if it satisfies the equation $\nabla S(g)=c \nabla V(g)$, where $\nabla$ denotes the gradient field and $c$ is the Einstein constant. Set $\tilde{S}=S-c\left(x_{1}^{d_{1}} x_{2}^{d_{2}}-1\right)$. The volume condition is given by $\partial \tilde{S} / \partial c=0$. Thus a $G$-invariant Einstein metric of volume one is a solution of the system

$$
\left.\begin{array}{l}
\frac{\partial \tilde{S}}{\partial x_{1}}=0 \\
\frac{\partial \tilde{S}}{\partial x_{2}}=0
\end{array}\right\}
$$

By (5.2), this is equivalent to

$$
\left.\begin{array}{r}
-\frac{d_{1}}{2 x_{1}^{2}}+\frac{t x_{2}}{2 x_{1}^{3}}-c d_{1} x_{1}^{d_{1}-1} x_{2}^{d_{2}}=0 \\
\frac{t-d_{2}}{2 x_{2}^{2}}-\frac{t}{4 x_{1}^{2}}-c d_{2} x_{1}^{d_{1}} x_{2}^{d_{2}-1}=0
\end{array}\right\}
$$

System (5.3) reduces to the polynomial equation

$$
2 t d_{1} x_{1}^{2}-2 d_{1} d_{2} x_{1}^{2}-t d_{1} x_{2}^{2}+2 d_{1} d_{2} x_{1} x_{2}-2 t d_{2} x_{2}^{2}=0
$$


Next, we need to find the nonzero number $t=[112]$. From Proposition 3.2, the space $G / K$ admits a unique Kähler-Einstein metric given by $x_{1}=1$ and $x_{2}=2$. Substituting these values into (5.4), we obtain the equation $2 t d_{1}-2 d_{1} d_{2}-4 t d_{1}+4 d_{1} d_{2}-8 t d_{2}=0$, from which we conclude that

$$
t=\frac{d_{1} d_{2}}{d_{1}+4 d_{2}} .
$$

We substitute this number into equation (5.4) and normalize by setting $x_{1}=1$. Then we obtain the equation

$$
d_{1} d_{2}\left(x_{2}-2\right)\left(d_{1} x_{2}+2 d_{2}\left(x_{2}-2\right)\right)=0,
$$

whose solutions are

$$
x_{2}=2 \quad \text { and } \quad x_{2}=\frac{4 d_{2}}{d_{1}+2 d_{2}} .
$$

The first solution determines a Kähler-Einstein metric, and the second solution determines a non-Kähler $G$-invariant Einstein metric on $G / K$.

Example 5.1. Consider the flag manifold $\operatorname{Sp}(\ell) / \mathrm{U}(p) \times \operatorname{Sp}(\ell-p)$ where $p=1$ and $\ell \geq 2$. Then we obtain the generalized flag manifold $M=\operatorname{Sp}(\ell) / \mathrm{U}(1) \times \operatorname{Sp}(\ell-1)$, which is the complex projective space $\mathbb{C} P^{2 \ell-1}$. The painted Dynkin diagram is

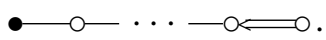

We see that $d_{1}=\operatorname{dim} \mathrm{m}_{1}=4(\ell-1)$ and $d_{2}=\operatorname{dim} \mathrm{m}_{2}=2$. Any $\operatorname{Sp}(\ell)$-invariant metric $\langle\cdot, \cdot\rangle$ on $\mathbb{C} P^{2 \ell-1}$ is determined by two positive parameters, $x_{1}$ and $x_{2}$, and has the form (5.1). According to Theorem 1.1 the non-Kähler $\operatorname{Sp}(\ell)$-invariant Einstein metric on $\mathbb{C} P^{2 \ell-1}$ is given by

$$
\langle\cdot, \cdot\rangle=\left.1(-B)\right|_{\mathfrak{m}_{1}}+\left.\frac{2}{\ell}(-B)\right|_{\mathfrak{m}_{2}} .
$$

The same result was also obtained by Ziller [Zil], using the method of Riemannian submersions.

5.2. The characterization of the constrained critical points. We will use a wellknown criterion (second-order partial derivatives) for minima, maxima, or saddle points of smooth functions to show that both Einstein metrics obtained in Theorem 1.1 are local minima of $\left.S\right|_{\mathcal{M}_{1}^{G}}$. We use the bordered Hessian $H$ of $S(g)$ restricted to the space $\mathcal{M}_{1}^{G}$ of $G$-invariant metrics with volume one. This is the $3 \times 3$ real symmetric matrix

$$
H=\left(\begin{array}{ccc}
0 & -\frac{\partial V}{\partial x_{1}} & -\frac{\partial V}{\partial x_{2}} \\
-\frac{\partial V}{\partial x_{1}} & \frac{\partial^{2} \tilde{S}}{\partial x_{1}^{2}} & \frac{\partial^{2} \tilde{S}}{\partial x_{1} \partial x_{2}} \\
-\frac{\partial V}{\partial x_{2}} & \frac{\partial^{2} \tilde{S}}{\partial x_{1} \partial x_{2}} & \frac{\partial^{2} \tilde{S}}{\partial x_{2}^{2}}
\end{array}\right),
$$


where $\tilde{S}=S-c\left(x_{1}^{d_{1}} x_{2}^{d_{2}}-1\right)$. Let det $H(g)$ be the determinant of the value $H(g)$ of $H$ at a critical point $g \in \mathcal{M}_{1}^{G}$. If $|H(g)|>0$ then $g$ is a local maximum of $\left.S\right|_{\mathcal{M}_{1}^{G}}$, while if $|H(g)|<0$ then $g$ is a local minimum of $\left.S\right|_{\mathcal{M}_{1}^{G}}$, and if $|H(g)|=0$ then $g$ is a saddle point (see $[\mathrm{MaT}])$.

Proof of Theorem 1.2. Since $V=x_{1}^{d_{1}} x_{2}^{d_{2}}$, we easily obtain

$$
-\frac{\partial V}{\partial x_{1}}=-d_{1} x_{1}^{d_{1}-1} x_{2}^{d_{2}} \quad \text { and } \quad-\frac{\partial V}{\partial x_{2}}=-d_{2} x_{1}^{d_{1}} x_{2}^{d_{2}-1} .
$$

From Equations (5.3), we also obtain

$$
\begin{aligned}
\frac{\partial^{2} \tilde{S}}{\partial x_{1}^{2}} & =\frac{d_{1}}{x_{1}^{3}}-\frac{3 t x_{2}}{2 x_{1}^{4}}-c d_{1}\left(d_{1}-1\right) x_{1}^{d_{1}-2} x_{2}^{d_{2}}, \\
\frac{\partial^{2} \tilde{S}}{\partial x_{2}^{2}} & =\frac{d_{2}-t}{x_{2}^{3}}-c d_{2}\left(d_{2}-1\right) x_{1}^{d_{1}} x_{2}^{d_{2}-2}, \\
\frac{\partial^{2} \tilde{S}}{\partial x_{1} \partial x_{2}} & =\frac{t}{2 x_{1}^{3}}-c d_{1} d_{2} x_{1}^{d_{1-1}} x_{2}^{d_{2}-1},
\end{aligned}
$$

where $t=d_{1} d_{2} /\left(d_{1}+4 d_{2}\right)$. We first examine the critical point $g=(1,2)$, that is, the Kähler-Einstein metric of $M$. A computation shows that

$$
|H(g)|=-\left(d_{1}+d_{2}\right) d_{1} d_{2} 2^{2 d_{2}-2}\left(\frac{d_{2}}{d_{1}+4 d_{2}}+c 2^{d_{2}}\right) .
$$

Since the Einstein constant $c$ and the dimensions $d_{1}, d_{2}$ are positive real numbers, it follows that $|H(g)|<0$, thus the Kähler-Einstein metric is a local minimum of $\left.S\right|_{\mathcal{M}_{1}^{G}}$. For the critical point $g=\left(1,4 d_{2} /\left(d_{1}+2 d_{2}\right)\right)$,

$$
\begin{aligned}
|H(g)|=- & d_{1}\left(\frac{4 d_{2}}{d_{1}+2 d_{2}}\right)^{2 d_{2}-2} \\
& \times\left(\frac{d_{1}^{3} d_{2}+5 d_{1}^{2} d_{2}^{2}+6 d_{1} d_{2}^{3}+2 d_{2}^{4}}{\left(d_{1}+2 d_{2}\right)\left(d_{1}+4 d_{2}\right)}+c d_{2}\left(\frac{4 d_{2}}{d_{1}+2 d_{2}}\right)^{d_{2}}\left(d_{1}+d_{2}\right)\right),
\end{aligned}
$$

so $|H(g)|<0$, and the $G$-invariant Einstein metric is also a local minimum of $\left.S\right|_{\mathcal{M}_{1}^{G}}$.

\section{Acknowledgements}

The second author wishes to thank Professor Yusuke Sakane for several useful discussions during his visit to the University of Patras. Both authors would like to thank the referee for his many important suggestions that led to improvements to this paper. 


\section{References}

[Ale] D. V. Alekseevsky, 'Homogeneous Einstein metrics', in: Differential Geometry and its Applications (Proceedings of the Conference) (J. E. Purkyně University, Brno, Czechoslovakia, 1987), pp. 1-21.

[AlA] D. V. Alekseevsky and A. Arvanitoyeorgos, 'Riemannian flag manifolds with homogeneous geodesics', Trans. Amer. Math. Soc. 359 (2007), 3769-3789.

[Arv] A. Arvanitoyeorgos, 'New invariant Einstein metrics on generalized flag manifolds', Trans. Amer. Math. Soc. 337 (1993), 981-995.

[ArC] A. Arvanitoyeorgos and I. Chrysikos, 'Motion of charged particles and homogeneous geodesics in Kähler C-spaces with two isotropy summands', Tokyo J. Math. 32 (2009), 487-500.

[Bes] A. L. Besse, Einstein Manifolds (Springer, Berlin, 1986).

[Bom] C. Böhm, 'Homogeneous Einstein metrics and simplicial complexes', J. Differential Geom. 67 (2004), 79-165.

[BWZ] C. Böhm, M. Wang and W. Ziller, 'A variational approach for compact homogeneous Einstein manifolds', Geom. Funct. Anal. 14 (2004), 681-733.

[BFR] M. Borderman, M. Forger and H. Römer, 'Homogeneous Kähler manifolds: paving the way towards new supersymmetric sigma models', Comm. Math. Phys. 102 (1986), 604-647.

[Bor] A. Borel, 'Kählerian coset spaces of semi-simple Lie groups', Proc. Natl. Acad. Sci. USA 40 (1954), 1147-1151.

[BoH] A. Borel and F. Hirzebruch, 'Characteristic classes and homogeneous spaces I', Amer. J. Math. 80 (1958), 458-538.

[DKe] W. Dickinson and M. M. Kerr, 'The geometry of compact homogeneous spaces with two isotropy summands', Ann. Global Anal. Geom. 34 (2008), 329-350.

[GOV] V. V. Gorbatzevich, A. L. Onishchik and E. B. Vinberg, Lie groups and Lie algebras. III. Structure of Lie Groups and Lie Algebras, Encyclopedia of Mathematical Sciences, 41 (Springer, Berlin, 1994).

[Hel] S. Helgason, Differential Geometry, Lie Groups and Symmetric Spaces (Academic Press, New York, 1978).

[Ikw] O. Ikawa, 'Motion of charged particles in Kähler C-spaces', Yokohama Math. J. 50 (2003), $31-39$.

[Ith] M. Itoh, 'On curvature properties of Kähler C-spaces', J. Math. Soc. Japan 30 (1978), 39-71.

[Kim] M. Kimura, 'Homogeneous Einstein metrics on certain Kähler C-spaces', Adv. Stud. Pure Math. 18-I (1990), 303-320.

[KoN] S. Kobayashi and K. Nomizu, Foundations of Differential Geometry, Vol. II (WileyInterscience, New York, 1969).

[LWa] C. LeBrun and M. Wang (eds), Surveys in Dierential Geometry Volume VI: Essays on Einstein Manifolds (International Press, Somerville, MA, 1999).

[MaT] J. E. Marsden and A. J. Tromba, Vector Calculus, 5th edn (W. H. Freeman, New York, 2003).

[NRS] Yu. G. Nikonorov, E. D. Rodionov and V. V. Slavskii, 'Geometry of homogeneous Riemannian manifolds', J. Math. Sci. 146 (2007), 6313-6390.

[Ohm] I. Ohmura, 'On Einstein metrics on certain homogeneous spaces', master's thesis (Japanese), Graduate School of Science, Osaka University, 1987 (unpublished).

[Sak] Y. Sakane, 'Homogeneous Einstein metrics on flag manifolds', Lobachevskii J. Math. 4 (1999), $71-87$.

[Wan] H. C. Wang, 'Closed manifolds with homogeneous complex structures', Amer. J. Math. 76 (1954), 1-32.

[WZ1] M. Wang and W. Ziller, 'On normal homogeneous Einstein manifolds', Ann. Sci. Éc. Norm. Supér. 18 (1985), 563-633.

[WZ2] M. Wang and W. Ziller, 'Existence and non-existence of homogeneous Einstein metrics', Invent. Math. 84 (1986), 177-194.

[Wol] J. A. Wolf, 'The action of a real semi-simple Lie group on a complex flag manifold, I: orbit structure and holomorphic arc components', Bull. Amer. Math. Soc. 75 (1969), 1121-1237.

[Zil] W. Ziller, 'Homogeneous Einstein metrics on spheres and projective spaces', Math. Ann. 259 (1982), 351-358. 
ANDREAS ARVANITOYEORGOS, Department of Mathematics, University of Patras, GR-26500 Rion, Greece

e-mail: arvanito@math.upatras.gr

IOANNIS CHRYSIKOS, Department of Mathematics,

University of Patras, GR-26500 Rion, Greece

e-mail: xrysikos@master.math.upatras.gr 